\title{
Cão naturalmente infectado por Trypanosoma evansi em Santa Maria, RS, Brasil
}

\author{
Dog naturally infected by Trypanosoma evansi in Santa Maria, RS, Brasil
}

\author{
Carina Franciscato ${ }^{\mathrm{I}}$ Sonia Terezinha dos Anjos Lopes ${ }^{\mathrm{II}}$ \\ Marta M. G. Teixeira ${ }^{\text {III }}$ Silvia Gonzalez Monteiro ${ }^{\text {IV }}$ \\ Patrícia Wolkmer ${ }^{\mathrm{V}}$ Bruna Carolina Garmatz $^{\mathrm{VI}}$ \\ Carlos Breno Paim ${ }^{\mathrm{VI}}$
}

\begin{abstract}
Descreve-se, neste trabalho, as alterações hematológicas e o proteinograma de um cão naturalmente infectado por Trypanosoma evansi. Este animal apresentou anemia normocítica normocrômica, leucopenia com linfopenia seguida por neutropenia e linfocitose; trombocitopenia; hiperproteinemia com aumento das frações beta e gama globulinas e hipoalbuminemia. Por ser este o primeiro relato de infecção por T. evansi em cães no município de Santa Maria, RS, destaca-se a sua importância epidemiológica, alertando os médicos veterinários para a existência de reservatórios do parasita na região e para a possibilidade de novos achados laboratoriais.
\end{abstract}

Palavras-chave: Trypanosoma evansi, cães, anemia, hipoalbuminemia,hipergamaglobulinemia.

\section{ABSTRACT}

This paper describes the hematological alterations and proteinogram of a dog naturally infected by Trypanosoma evansi. This dog was presented with normochromic-normocytic anemia, leucopenia with lymphopenia followed for neutrophenia and lymphocitosis; and trombocitopenia. Hyperproteinemia with an increase of beta and gamma globulin fractions and hypoalbuminemia. By being the first case reported of T. evansi infection in dogs in Santa Maria, RS, Brazil, the epidemiological significance of such findings will alert the veterinarians to the existence of a possible parasite's reservoir in the region warning to the possibility of new laboratory findings.
Key words: $\begin{gathered}\text { Trypanosoma evansi, dogs, anemia, } \\ \text { hypoalbuminemia, hypergamaglobulinemia. }\end{gathered}$

Trypanosoma evansi é um protozoário hemoflagelado da família Trypanosomatidae, transmitido mecanicamente por moscas hematófagas dos gêneros Stomoxys sp. e Tabanus sp. (HOARE, 1972). No Brasil, foram descritas duas formas da doença causada por T. evansi: a síndrome aguda, que causa morte rápida em eqüinos e cães não-tratados, e a crônica, que afeta diversos animais silvestres, principalmente capivaras (Hydrochaeris hydrochaeris) e quatis (Nasua nasua) (HERRERA et al., 2005). Os cães podem apresentar perda de peso, fraqueza progressiva, inapetência e anemia (AQUINO et al., 1999). Além disso, animais parasitados também podem apresentar febre intermitente, conjuntivite, edema das pernas e porções inferiores e aumento dos linfonodos superficiais (LEVINE, 1973).

O presente estudo tem como objetivo comunicar o achado de T. evansi em esfregaços sangüíneos de um cão naturalmente infectado, além de mostrar as alterações hematológicas e o proteinograma apresentados pelo animal. Este trabalho possui importância epidemiológica por ser o primeiro caso de

IPrograma de Pós-graduação em Medicina Veterinária, Universidade Federal de Santa Maria (UFSM), RS 509, Km 05, no 3112, 97110-620, Santa Maria, RS, Brasil. E-mail: carinafranciscato@yahoo.com.br. Autor para correspondência.

IIDepartamento de Clínica de Pequenos Animais, UFSM, Santa Maria, RS, Brasil.

IIIDepartamento de Parasitologia, Instituto de Ciências Biomédicas, Universidade de São Paulo (USP), São Paulo, SP, Brasil.

${ }^{\mathrm{IV}}$ Departamento de Parasitologia, UFSM, Santa Maria, RS, Brasil.

vautônomo, Santa Maria, RS, Brasil.

${ }^{\mathrm{VI} C u r s o}$ de Medicina Veterinária, UFSM, Santa Maria, RS, Brasil.

${ }^{\mathrm{VII} H o s p i t a l}$ Veterinário, UFSM, Santa Maria, RS, Brasil. 
T. evansi em cães identificado no Laboratório de Análises Clínicas do Hospital de Clínicas Veterinárias (HCV) da Universidade Federal de Santa Maria (UFSM).

Um cão sem raça definida, macho, de 3 anos de idade, residente no município de Santa Maria e esporadicamente levado para propriedades rurais deste município, foi recebido no HCV da UFSM apresentando perda de peso há um ano, aproximadamente, fraqueza progressiva, inapetência e anemia. Sinais clínicos semelhantes foram descritos em eqüinos (SILVA et al., 1995) e em cães (AQUINO et al., 1999) infectados por T. evansi.

Durante a execução do hemograma, verificou-se a presença de protozoários do gênero Trypanosoma (Figura 1a); esfregaços confeccionados da capa flogística mostraram uma maior concentração de protozoários. A análise morfológica dos tripanossomas encontrados no cão estudado foi sugestiva de $\boldsymbol{T}$. evansi devido à ausência de cinetoplasto nestes flagelados. A coloração dos esfregaços com o corante de DNA fluorescente 4'-6'diamidino-2-phenylindole (DAPI) confirmou a ausência de moléculas de kDNA, uma característica exclusiva de T. evansi compartilhada por todos os isolados brasileiros já estudados (VENTURA et al., 2000). Os tripanossomas foram também confirmados como $\boldsymbol{T}$. evansi utilizando-se um método de PCR espécieespecífico desenvolvido por VENTURA et al. (2002), que tem como base o fragmento Te664 como seqüência repetitiva em cromossomos de $\boldsymbol{T}$. evansi.

No eritrograma (Tabela 1), evidenciou-se uma anemia normocítica normocrômica, diferindo de SILVA et al. (1995), que citam a presença de anemia microcítica hipocrômica em cães naturalmente infectados por T. evansi. Provavelmente a anemia apresentada pelo animal do presente relato representa uma resposta à doença infecciosa crônica. SILVA et al. (1995) propõem algumas causas de anemia na Tripanossomíase Africana como: hemólise extra e intravascular pelo sistema imune, efeito traumático direto do tripanossoma ou eritropoiese diminuída.

No leucograma (Tabela 1), verificou-se leucopenia causada por linfopenia e eosinopenia, mas um novo hemograma revelou neutropenia e linfocitose, com leucócitos totais dentro da normalidade. O discreto aumento de linfócitos em cães experimentalmente infectados por T. evansi foi atribuído, por RUE et al. (2000), à resposta do hospedeiro infectado. Observou-

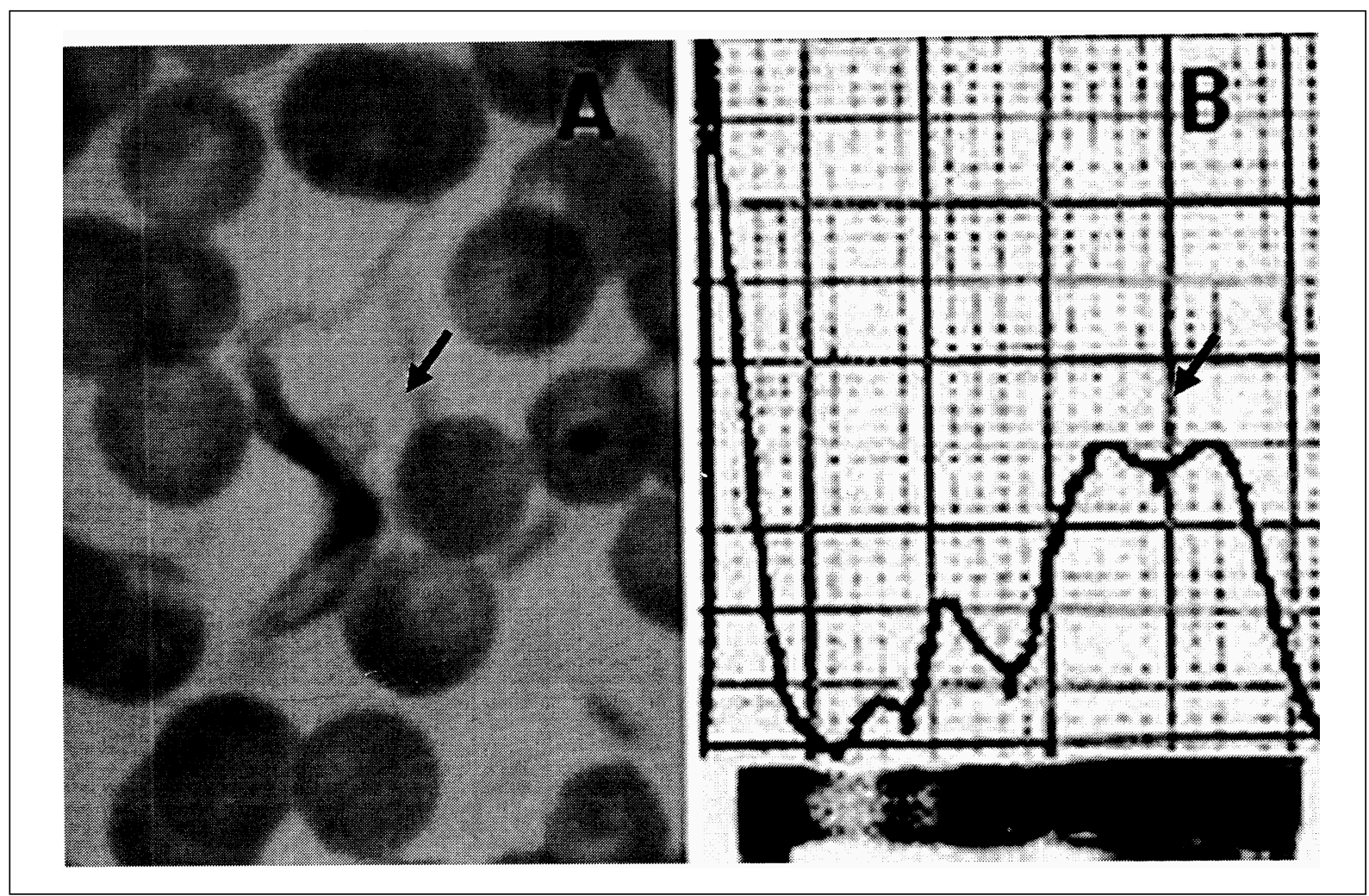

Figura 1 - Esfregaço sangüíneo e representação gráfica da migração de proteínas de um cão infectado por Trypanosoma evansi. a) Trypanosoma evansi detectado no esfregaço sangüíneo corado com panótico rápido, visualizado em um aumento de 1000 vezes. b) Representação gráfica da migração das proteínas séricas caracterizando uma gamopatia policlonal, evidenciada por um pico de base larga (seta). 
Tabela 1 - Parâmetros hematológicos e bioquímicos do cão naturalmente infectado por T. evansi.

\begin{tabular}{lllr}
\hline Parâmetro & \multicolumn{1}{c}{$1^{\mathrm{a}}$} & \multicolumn{1}{c}{$2^{\mathrm{a}}$} & \multicolumn{1}{c}{$\begin{array}{c}\text { Valores de } \\
\text { referência }\end{array}$} \\
\hline Hemáciação & 4,44 & 4,05 & $5.5-8.5\left(\mathrm{x} 10^{6} / \mu \mathrm{l}\right)^{\mathrm{a}}$ \\
Hemoglobina & 10,5 & 10,1 & $12-18(\mathrm{~g} / \mathrm{dl})^{\mathrm{a}}$ \\
Hematócrito & 32 & 29 & $37-55(\%)^{\mathrm{a}}$ \\
VCM* $^{*}$ & 72,1 & 71,6 & $60-77(\mathrm{fl})^{\mathrm{a}}$ \\
CHCM** $^{*}$ & 32,8 & 34,8 & $32-36(\%)^{\mathrm{a}}$ \\
PPT*** $^{*}$ & 10,4 & 9,8 & $6,0-8,0 \mathrm{~g} / \mathrm{dL}^{\mathrm{a}}$ \\
Leucócitos totais & 5.400 & 9.300 & $6.000-17.000 / \mu \mathrm{L}^{\mathrm{a}}$ \\
Neutrófilos & 4.212 & 2.604 & $3.000-11.500 / \mu \mathrm{L}^{\mathrm{a}}$ \\
Linfócitos & 540 & 5.580 & $1.000-4.800 / \mu \mathrm{L}^{\mathrm{a}}$ \\
Monócitos & 648 & 372 & $150-1.350 / \mu \mathrm{L}^{\mathrm{a}}$ \\
Eosinófilos & 0 & 744 & $150-1.250 / \mu \mathrm{L}^{\mathrm{a}}$ \\
Plaquetas & - & 103.500 & $200-500 \mathrm{x} 10^{3 \mathrm{a}}$ \\
Proteínas totais & - & 8,7 & $5,4-7,1(\mathrm{~g} / \mathrm{dl})^{\mathrm{b}}$ \\
Albumina & - & 2,4 & $2,6-3,3(\mathrm{~g} / \mathrm{dl})^{\mathrm{b}}$ \\
Alfa 1 Globulina & - & 0,2 & $0,2-0,5(\mathrm{~g} / \mathrm{dl})^{\mathrm{b}}$ \\
Alfa 2 Globulina & - & 0,9 & $0,3-1,1(\mathrm{~g} / \mathrm{dl})^{\mathrm{b}}$ \\
Beta Globulina & - & 2,9 & $0,7-1,3(\mathrm{~g} / \mathrm{dl})^{\mathrm{b}}$ \\
Gama Globulina & - & 2,4 & $0,5-1,3(\mathrm{~g} / \mathrm{dl})^{\mathrm{b}}$ \\
\hline
\end{tabular}

*Volume corpuscular médio.

**Concentração média de hemoglobina.

***Proteína plasmática total.

${ }^{a}$ MEINKOTH \& CLINKENBEARD (2000).

${ }^{\mathrm{b}} \mathrm{KANEKO}$ et al. (1997).

se ainda a presença de linfócitos reativos e trombocitopenia. RUE et al. (1997) descreveram diminuição do número de plaquetas em cães experimentalmente infectados por T. evansi e JAIN (1993) cita que, em cães parasitados, a trombocitopenia ocorre pela diminuição da sobrevida das plaquetas.

No proteinograma do animal (Tabela 1), encontrou-se hipoalbuminemia, aumento de beta globulinas e hipergamaglobulinemia, com características de gamopatia policlonal (Figura 1b), que pode ser justificada pela produção de imunoglobulinas em resposta à estimulação antigênica causada pelo parasita circulante (TIZARD, 2002). SANDOVAL et al. (1994) também observaram hipoalbuminemia e hipergamaglobulinemia na eletroforese sérica de ratos experimentalmente infectados.

A avaliação radiológica torácica do animal revelou aumento generalizado da silhueta cardíaca e congestão pulmonar. Uma semana após o tratamento com digitálico, diurético e antibiótico (cefalosporina), indicado devido aos sinais de insuficiência cardíaca congestiva, não foi mais possível a observação de tripanossomas nos esfregaços sangüíneos. Passados três meses, o cão retornou ao HCV para nova avaliação. Nesta ocasião, o proprietário relatou que havia usado tetraciclina no animal e, em novos esfregaços sangüíneos, não foram encontrados parasitas. Desta forma, apesar de não ter sido tratado com aceturato de diminazeno, que é a droga usada para o tratamento de T. evansi (COLPO et al., 2005), o cão se recuperou da infecção. Este comportamento é bastante diferente da maioria dos casos descritos em cães, sendo as infecções por T. evansi em cães e eqüinos geralmente fatais, se não tratadas rápida e adequadamente (MAHMOUD \& GRAY, 1980).

No Brasil, o achado de animais infectados por T. evansi é predominante na região do Pantanal e em áreas vizinhas. Os animais encontrados infectados fora desta região sempre apresentaram um histórico ligado ao Pantanal. Nesta região, a infecção por $\mathbf{T}$. evansi é uma enzootia em equilíbrio, com raros casos de surtos em eqüinos e cães (SILVA et al., 1995; HERRERA et al., 2004). Apenas muito recentemente foram relatados casos de infecção aguda e grave por $\boldsymbol{T}$. evansi, com a morte de vários animais sem histórico de passagem pela região do Pantanal, no Estado do Rio Grande do Sul: cães em Uruguaiana, fronteira oeste do RS (COLPO et al., 2005), e eqüinos em São Sepé (região central do RS), que, provavelmente, foram infectados no município de Alegrete, na fronteira oeste do RS (CONRADO et al., 2005), sendo importante ressaltar que dezenas de eqüinos com tripanossomíase por $\boldsymbol{T}$. evansi morreram no Estado entre 2003 e 2004 (RODRIGUES et al., 2005). Estes relatos, em conjunto com este trabalho, indicam que T. evansi deve ter sido recentemente introduzido na região Sul do Brasil, sendo provavelmente originário da Argentina, onde é comum em eqüinos (MONZON et al., 1995).

\section{REFERÊNCIAS}

AQUINO, L.P.C.T. et al. Clinical, parasitological and immunological aspects of experimental infection with Trypanosoma evansi in dogs. Mem Inst Oswaldo Cruz, Rio de Janeiro, v.94, p.255-260, 1999.

COLPO, C.B. et al. Infecção natural por Trypanosoma evansi em cães. Ciência Rural, Santa Maria, v.35, n.3, p.717-719, 2005.

CONRADO, A.C. et al. Infecção natural por Trypanosoma evansi em cavalos na região central do Estado do Rio Grande do Sul. Ciência Rural, Santa Maria, v.35, n.4, p.928-931, 2005.

HERRERA, H.M. et al. Enzootiology of Trypanosoma evansi in Pantanal, Brazil. Vet Parasitol, v.125, p.263-275, 2004.

HERRERA, H.M. et al. Domestic and wild mammals infection by Trypanosoma evansi in a prisitine area of the Brazilian Pantanal region. Parasitol Res, v.96, n.2, p.121-126, 2005.

HOARE, C.A. The salivaria, subgenus Trypanozoon. In: The Trypanosomoses of mammals: a zoological

Ciência Rural, v.37, n.1, jan-fev, 2007. 
monography. Oxford: Blackwell Scientific Publications, 1972. p.476-604.

JAIN, N.C. Essentials of veterinary hematology. Philadelphia: Lea \& Febiger, 1993. 417p.

KANEKO, J.J. et al. Appendixes. In: Clinical biochemistry of domestic animals. 5.ed. London: Academic, 1997. p.885-905. Apêndice IX.

LEVINE, N.D. Protozoan parasites of domestic animals and of man. 2.ed. Minneapolis: Burguess, 1973. 406p.

MAHMOUD, M.M.; GRAY, A. Trypansosomiasis due to Trypanosoma evansi (Steel, 1885) Balbiani,1888. A review of recent research. Trop Anim Hlth Prod, v.12, p.35-47, 1980

MEINKOTH, J.H.; CLINKENBEARD, K.D. Normal hematology of the dog. In: FELDMAN, B.F. et al. Schalm's veterinary hematology. 5.ed. Philadelphia: Lippincott Williams \& Wilkins, 2000. Cap.163, p.1057-1063.

MONZON, C.M. et al. Outbreaks of equine trypanosomiasis caused by Trypanosoma evansi in Formosa Province, Argentina. Vet Sci Tech, v.14, n.3, p.747-52, 1995.

RODRIGUES, A. et al. Surtos de Tripanossomíase por Trypanosoma evansi em eqüinos no Rio Grande do Sul: aspectos epidemiológicos, clínicos, hematológicos e patológicos. Pesq Vet Bras, v.25, n.4, p.239-249. 2005

RUE, M.L. et al. Coagulopathy in dogs infected with Trypanosoma (Trypanozoon) evansi (Steel, 1885)
Balbiani,1888. Parasitología al día, v.21, n.3-4, p.92-96, 1997.

RUE, M.L. et al. Leukocytes and reticulocytes counts in acute infection of dogs with Trypanosoma evansi (Steel, 1885) Balbiani, 1888. Rev Latinoamer de Microbiol, v.42, p.163166, 2000.

SANDOVAL, G.L. et al. Alterações bioquímicas e histopatológicas de um cão e ratos infectados com Trypanosoma evansi. A Hora Veterinária, v.14, n.81, p.53-55, 1994.

SILVA, R.A.M.S. et al. Pathogenesis of Trypanosoma evansi in dogs and horses: hemathological and clinical aspects. Ciência Rural, Santa Maria, v.25, p.233-238, 1995.

TIZARD, I.R. Imunologia veterinária: uma introdução. 6.ed. São Paulo: Roca, 2002. 532p.

VENTURA, R.M. et al. Molecular and morphological studies of Brazilian Trypanosoma evansi stocks: the total absence of kDNA in trypanosomes from both laboratory stocks and naturally infected domestic and wild mammals. J Parasitol, v.86, n.6, p.1289-1298, 2000.

VENTURA, R.M. et al. Genetic relatedness among Trypanosoma evansi stocks by random amplification of polymorphic DNA and evaluation of a synapomorphic DNA fragment for species-specific diagnosis. Int J Parasitol, v.32, n.1, p.53-63, 2002. 